\title{
Preface to the empirical software engineering special issue on selected papers from RE'19
}

\author{
Kelly Blincoe ${ }^{1} \cdot$ Daniela Damian $^{2} \cdot$ Anna Perini $^{3}$ \\ Published online: 6 October 2020 \\ (C) Springer Science+Business Media, LLC, part of Springer Nature 2020
}

Welcome to this special issue that includes selected empirical studies in Requirements Engineering (RE).

The selected studies extend research presented at RE'19, the 27th IEEE International Requirements Engineering Conference. The RE conference is the premier international forum for researchers, practitioners, educators, and students to present and discuss the most recent innovations, experiences, and concerns in the discipline of requirements engineering. RE'19 had the theme " $R E$ and Collective Intelligence in the Days of $A I$ ' and was held in Jeju Island, South Korea from September 23-27, 2019 (Damian et al. 2019).

Four papers employing empirical methods stood out at RE'19 as exceptional contributions. The authors of these candidate papers were invited to submit extended manuscripts for this special issue. The submitted manuscripts were each rigorously peer-reviewed by three reviewers, and three were accepted for inclusion in this special issue.

Summary of the Papers The first article, "Are there gender differences when interacting with social goal models? A quasi-experiment” by Catarina Gralha, Miguel Goulão, and João Araújo, aims to understand the impact of different cognitive styles on interacting with iStar 2.0 models, which are used to model software requirements. The manuscript describes a study with 180 participants, where the participants created, modified, and reviewed iStar 2.0 models and accuracy, speed, and ease was measured. The study collected data using eye-tracking, EEG and EDA sensors, and participants' feedback. The study found that participants with cognitive styles frequently seen in women solved the tasks with lower speed but higher accuracy while participants with cognitive styles frequently seen in men were better at identifying relevant information. The results show the importance of diversity in software teams.

Kelly Blincoe

k.blincoe@auckland.ac.nz

Department of Electrical, Computer, and Software Engineering, University of Auckland, 5 Grafton Road, Auckland 1010, New Zealand

2 Department of Computer Science, University of Victoria, 3800 Finnerty Road, Victoria, BC V8P $5 \mathrm{C} 2$, Canada

3 Fondazione Bruno Kessler, Via Sommarive, 18 - POVO, 38123 Trento, Italy 
The second article, "Feature Requests-based Recommendation of Software Refactorings" by Ally S. Nyamawe, Hui Liu, Nan Niu, Qasim Umer, and Zhendong Niu, proposes a new learning-based approach to recommend software refactorings that can enable software systems to adapt to new requirements. The approach was evaluated on 55 open source Java projects, and the results indicate the recommended refactorings achieve high accuracy. The approach can help software projects respond more quickly to new or changing requirements.

The third article, "Automated Demarcation of Requirements in Textual Specifications: A Machine Learning-Based Approach" by Sallam Abualhaija, Chetan Arora, Mehrdad Sabetzadeh, Lionel C. Briand, and Michael Traynor, proposes an automated approach for demarcating requirements in free-form requirements specifications and develops a prototype tool, DemaRQ, based on the new approach. Through an empirical study with 33 industrial requirement specifications and a user study with software requirements experts, the approach and associated prototype tool were found to be accurate and useful to practitioners. This new approach and its associated tool can help requirements engineers quickly determine which statements in a requirements specification represent requirements.

Collectively, these three contributions represent diverse and relevant examples of empirical research in RE. They all aim to assess the quality and maturity of proposed solutions, an essential step to foster further research and progress towards adoption by practitioners.

Acknowledgements We thank the authors for their excellent manuscripts. We are deeply grateful to the reviewers of this special issue for their time and constructive feedback, which helped to shape the articles. We are also thankful to the Empirical Software Engineering journal and the Editors-in-Chief, Robert Feldt and Thomas Zimmermann, for their support throughout the process of preparing this special issue.

\section{Reference}

Damian DE, Perini A, Lee S-W (2019) 27th IEEE International Requirements Engineering Conference, RE 2019, Jeju Island, Korea (South), September 23-27, 2019. IEEE 2019, ISBN 978-1-7281-3912-8

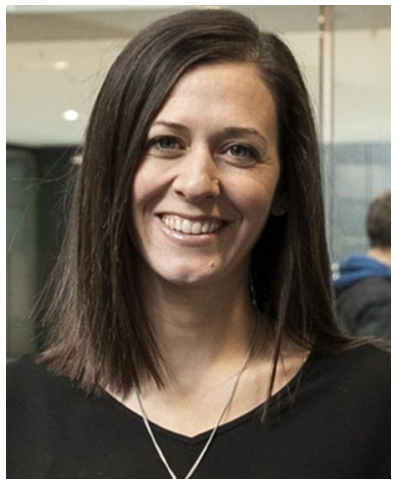

Kelly Blincoe is a Senior Lecturer at the University of Auckland's Department of Electrical, Computer, and Software Engineering, where she leads the Human Aspects in Software Engineering Lab (HASEL, https://hasel. auckland.ac.nz/). Her research is mainly in the human aspects of software engineering with a focus on collaborative software development and software requirements. She currently serves on the editorial board of the Empirical Software Engineering Journal and the Journal of Systems and Software. She is also on the Executive Board of Software Innovation New Zealand. 


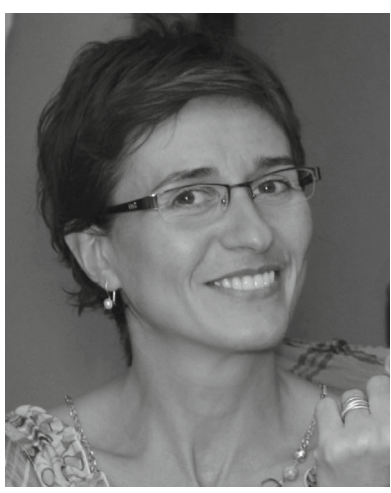

Daniela Damian is a Professor of Software Engineering in University of Victoria's Department of Computer Science, where she leads research in the Software Engineering Global interAction Laboratory (SEGAL, thesegalgroup.org/people/daniela-damian). Her research interests include Software Engineering, Requirements Engineering, Empirical Software Engineering and Global Software Development. Her recent work has studied developers' socio-technical coordination in geographically distributed software projects, as well as stakeholder management in large software ecosystems. Daniela has served on the program committee boards or Program CoChair of several software engineering conferences, as well as on the editorial boards of Transactions on Software Engineering and the Journal of Requirements Engineering. She is serving on the Advisory Board at the Empirical Software Engineering Journal and as the Human Aspects Area Editor for the Journal of Software and Systems.

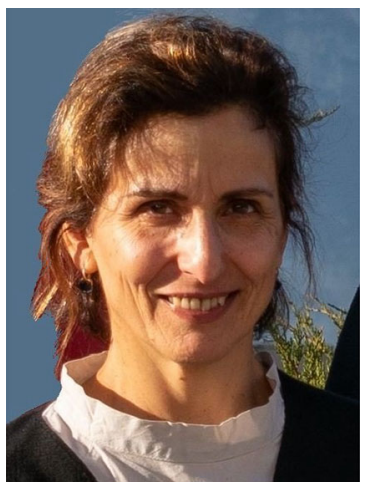

Anna Perini is a senior researcher at the Software Engineering research unit of Fondazione Bruno Kessler - ICT, Trento (Italy), where she is leading research in Requirements Engineering. She teaches Requirements Engineering at the University of Trento, MSc degree in Computer Science. Her research interests include requirements engineering, agent-oriented software development methodologies, conceptual modelling, decision making in requirements engineering, and empirical studies. She has served as program chair and program committee member of several international conferences and workshops, and regularly reviews papers for top journals in software engineering. 